the experimenter about what constitutes an acceptable stimulus range in a laboratory experiment, or to different levels of anxiety that men and women bring into the pain laboratory (cf. Rollman 1995).

Obviously, if the outcome of the present re-analysis were representative of similar studies, it would deepen rather than reduce the gap between the "inductive" and "deductive" approaches to sex differences in pain that have been so convincingly pursued by BERKLEY. In contrast to traditional scaling or threshold approaches, however, which result in unknown mixtures of sensory and judgmental effects, signal-detection methodology might clarify at what psychological level sex differences in pain will have to be conceptualized.

\section{Psychobiological sex differences in pain: Psychological as much as biological}

\section{K. Gijsbers ${ }^{a}$ and C. A. Niven \\ aDepartment of Psychology and ${ }^{b}$ Department of Nursing, Stirling University, Stirling FK9 4LA, Scotland. kjg1@stir.ac.uk}

\begin{abstract}
The argument of BERKLEY for the existence sex differences in pain is based on biological factors. We suggest that the psychological
\end{abstract} evidence for such differences is more substantial.

Overall, BERKLEY is skeptical about the behavioural ("inductive") evidence for a sex difference in pain sensitivity. She prefers to weight her argument for the existence of such differences on socalled "deductive" evidence of a primarily biological nature, arguing persuasively that there is a physiological basis for differences between the sexes in pain sensitivity. Thus, BERKLEY apparently concludes that there is only weak behavioural evidence for sex differences in pain despite the presence of a number of biological factors which should predispose us in that direction. We would argue that she underemphasises the behavioural evidence, so well reviewed in her target article.

Female hyperalgesia, demonstrated under laboratory conditions, is remarkably consistent with the evidence for sex differences in the occurrence of a range of clinical pain. The fact that much of this evidence is incidental to the main purpose of the surveys involved would seem to increase rather than decrease the significance of the data (sect. 2, para. 1). Moreover, although it is undoubtedly true that sex differences in clinical pain interact with "situational, temporal, attitudinal, and social factors," these psychosocial factors may in themselves be subject to sex differences, which act either to exaggerate or minimise the effects of physiological differences. For example, the greater use of behavioural coping strategies by women (Jensen et al. 1994) may reduce female pain experience just as the greater readiness of male physicians to diagnose angina in men may increase the reporting of chest pain in males.

In contrast to her skepticism about the human data, BERKLEY seems sympathetic to the evidence for sex differences in pain sensitivity in nonhuman species. But these responses might likewise be modulated by differences in emotions and behaviours provoked in male and female animals by the testing procedures rather than straightforward demonstrations of fundamental physiological differences in nocicepitive mechanisms.

BERKLEY's focus on physiological mechanisms and on deductive analysis has the unfortunate effect of distracting attention from the psychological mechanism which may be an important source of sex differences. This can be seen most clearly in the analysis of sex differences related to the reproductive organs and to sex hormones and their temporal features. Whereas we would agree with BERKLEY that many of the observed sex differences in pain are psychobiological consequences of the specific role of women in reproduction, we feel that these consequences are as much "psycho" as "bio" in nature. BERKLEY emphasises the possi- bility that the regular experience of menstrual pain in otherwise healthy young women could result in the sensitisation of nocioceptive mechanisms - a conclusion with which we would readily concur (Gijsbers \& Niven 1993). However, some of our recent findings would point to the beneficial effects of previous pain experience, in that we found that women in childbirth utilise a range of behavioural and mental strategies which they have effectively exercised during previous painful experiences (Niven \& Gijsbers 1996). This psychological effect may offset physiological "disadvantages."

BERKLEY does discuss one major psychological mechanism in her review of temporal conditioning, but we wonder what evidence would support the kind of learned time-locked nociception which Berkley relates to the periodic hormonal variations experienced by women. Most women with regular menstrual cycles have diminished levels of menstrual pain after giving birth to their first child. Why should this be the case if it is the cycling sex hormones that are providing "discriminative stimuli for conditioning" (sect. 3, para. 6)? What is needed in this context is a long-term behavioral study of pain in women, which encompasses menstruation, pregnancy, parturition, post-natal menstruation, and menopause.

BERKLEY's target article is a useful contribution to the understanding of biological mechanisms that might (we emphasise "might") underlie sex differences in pain perception. In agreeing with the appropriateness of her quotation from Irigeray (1993) regarding the critical importance of the issues she addresses, we feel that she undervalues the adage that the proper study of woman lies in the study of women. Only through such study will we come to understand the extent to which individual differences in suffering are dependent on generalisable sex differences. It is these differences in the experience of clinical pain which are of critical importance in the recommendations for treatment.

The complexity of the interactions between the biological and psychosocial factors involved in pain perception, report, and response are such that we would echo BERKLEY's conclusion that it is as yet inappropriate to call for "different overall treatment regimens for females and males" (sect. 2, last para.). Equality of treatment is much more important, and achieving it remains a significant problem (Niven \& Carroll 1993). However, we would base our conclusions not on the insignificance of sex differences in behaviour and perception but on their complexity.

\section{Persistent pain: Trim the branches or fell the tree?}

\section{Richard H. Gracely}

Pain and Neurosensory Mechanisms Branch, National Institute of Dental Research, National Institutes of Health, Bethesda, MD 20892. gracely@yoda.nidr.nih.gov

Abstract: In patients with pain characterized by a painful focus and allodynia, the painful symptoms arise from altered central processing that is initiated and subsequently maintained by persistent input from nociceptive afferents. Treatments directed at this normal consequence of persistent input are inherently limited. The most efficacious treatments will target the pathology, the various sources of ongoing nociceptor input. [BLUMBERG et al.; CODERRE \& KATZ; DICKENSON]

Both clinical and experimental studies provide converging lines of evidence for endogenous processes that both exacerbate and attenuate pain. While a host of animal models have furthered our understanding, it is important to focus on evidence from the ultimate target of this research, the underlying mechanisms of intractable pain syndromes.

BLUMBERG et al. discuss differences in patients with sympathetic involvement; one group presents with warm swollen extremities, another group presents with spontaneous pain, allodynia, and often an identifiable pain focus associated with 